\title{
Invasive Thyroid Angiosarcoma With a Favorable Outcome
}

\author{
Joana Couto ${ }^{1, *}$; Raquel G Martins ${ }^{1}$; Ana Paula Santos ${ }^{1}$; Joana Matos ${ }^{2}$; Isabel Torres ${ }^{1}$ \\ ${ }_{1}^{1}$ Endocrinology Department, Portuguese Institute of Oncology FG, Porto, Portugal \\ 2 Pathology Department, Portuguese Institute of Oncology FG, Porto, Portugal \\ ${ }^{*}$ Corresponding author: Joana Couto, Endocrinology Department, Portuguese Institute of Oncology FG, Porto, Portugal. Tel: + 351-969014901, Fax: + 351-225084001, E-mail: joanam- \\ couto@hotmail.com
}

Received: October 30, 2013; Revised: May 31, 2014; Accepted: June 13, 2014

\begin{abstract}
Introduction: Thyroid angiosarcoma is a malignant neoplasm, which usually shows local aggressive behavior and associated with a high recurrence rate. It was originally described in the Alpine region and extremely rare in other parts of the world.

Case Presentation: An asymptomatic 61-year-old woman presented with a right lobe thyroid nodule with fine needle aspiration cytology indicating "suspicious for malignancy" was reported in our study. Histological examination revealed a vascular $35 \mathrm{~mm}$ neoplasm with areas of necrosis. Immunohistochemistry staining had negative results for MNF, CAM5.2, CD34, thyroglobulin, and positive for CD31. The findings described were compatible with angiosarcoma diagnosis. Besides, a $4 \mathrm{~mm}$ papillary microcarcinoma was found in the left lobe. Computerized tomography (CT) scan performed about two months after the operation, showed a right neck nodular lesion, conditioning tracheal deviation. At our institution, the study performed was consistent with local recurrence (angiosarcoma). Tumor excision was performed and invasion to larynx, trachea and esophagus was detected intraoperatively. Histopathologic examination confirmed tumor recurrence and the patient was submitted to radiotherapy (60 Gy), completed four years ago. There is not, so far, any evidence of tumor recurrence.

Conclusions: We described a rare case of a usually aggressive thyroid angiosarcoma in a patient living in a non-Alpine region, with an unusual favorable outcome after the operation and radiotherapy.
\end{abstract}

Keywords:Angiosarcoma; Thyroid; Neoplasia

\section{Introduction}

Most thyroid tumors are primary and of epithelial origin, while tumors of mesenchymal origin, common in other organs, rarely occur in this gland (1-3). Vascular thyroid lesions include benign neoformation such as hemangiomas, and malignant tumors, like angiosarcoma and angiosarcomatoid carcinoma. Angiosarcoma is a rare soft tissue tumor, accounting for about $1-2 \%$ of all sarcomas, mainly occurring in the skin and head and neck superficial soft tissues $(1,4,5)$. Thyroid angiosarcoma is a rare malignant tumor of endothelial differentiation, mainly found in European mountainous regions of the Alps (Switzerland, Austria and Northern Italy), where it represents up to $16 \%$ of thyroid malignancies $(1,5-8)$. Its high prevalence in those regions is thought to be due to iodine deficiency associated goiter, because most thyroid angiosarcomas occur in a multinodular gland $(6,7)$. Although its etiology remains unknown, it was suggested that endothelial proliferation after recurrent hemorrhage in nodules within a long-standing multinodular goiter, can induce neoplastic transformation $(1,2)$. It is more common in females (ratio 9:3) and mainly occurs between the fifth and eighth decades (the median age of 65 years) $(1,6,9)$. Generally, these tumors present as a painless infiltrating mass with tendency to increase its size (1), which can vary from 2.5 to $11 \mathrm{~cm}$, being usually unifocal $(6,10)$. Cytological characteristics of this type of cancer have not been clearly defined yet and are rarely described in the literature (5). Histological examination often reveals extensive areas of necrosis, hemorrhage with the presence of anastomosing channels lined by endothelial cells. Endothelial differentiation is evidenced by the expression of vascular markers such as CD31 (the most sensitive and specific marker), CD34 and factor VIII related antigen. In many cases, there may be coexpression of cytokeratins. Immunonegativity for thyroglobulin supports its diagnosis (6-8). Some authors believe that most thyroid angiosarcomas are in fact anaplastic thyroid carcinomas with angiomatoid features; however, this distinction is considered academic, since their prognosis and treatment are similar $(1,8,11)$. Treatment of these tumors is conditioned by local aggressive behavior and high recurrence rate, consisting of radical surgery wherever feasible. Radiation therapy seems to be effective in some patients and can be complemented with chemotherapy using Adriamycin $(1,7)$. Recurrence and development of metastasis are common, even after complete resection (2) and the prognosis is poor, due to local aggressive behavior. Presence of distant metastasis

Copyright (C) 2014, Research Institute For Endocrine Sciences and Iran Endocrine Society; Published by Kowsar. This is an open-access article distributed under the terms of the Creative Commons Attribution-NonCommercial 4.0 International License (http://creativecommons.org/licenses/by-nc/4.0/) which permits copy and redistribute the material just in noncommercial usages, provided the original work is properly cited. 
and extracapsular tumor extension are frequently associated with a poor prognosis (1). Metastasis is commonly found in local lymph nodes, lung and bone marrow $(1,12)$. Mean 5-year survival rate is near 33\% and most patients die in less than six months, regardless of treatment $(1,6)$.

\section{Case Presentation}

We present a 61-year-old-women with no relevant medical history, born in northern Portugal, where she has always lived. When a neck mass was noticed, neck ultrasound was performed showing two solid thyroid nodules, one in the right lobe with about $3 \mathrm{~cm}$ and another in the left lobe with about $9 \mathrm{~mm}$. There were no associated compression symptoms. Fine needle aspiration (FNA) cytology was held reporting "suspicious for malignancy". The patient underwent total thyroidectomy and histological examination showed a $35 \mathrm{~mm}$ heterogeneous and hemorrhagic vascular neoplasm with dilated vessels, areas of necrosis, with swollen endothelial cells and atypical mitoses between thyroid follicles and cells. Immunohistochemistry staining showed negative results for cytokeratins MNF, CAM5.2, $\mathrm{CD} 34$, thyroglobulin and positive finding for CD31. These findings confirmed the diagnosis of angiosarcoma. A 4 $\mathrm{mm}$ papillary microcarcinoma was also identified in the left lobe, without any poor prognosis criteria. Neck computerized tomography (CT) scan performed two months after the operation showed a $35 \mathrm{~mm}$ nodular lesion in the right surgical bed, with irregular margins, conditioning tracheal deviation. The patient was referred to our institution. FNA of the nodule was held showing local recurrence of the previously diagnosed malignancy. 18F-FDG PET-CT identified a single neck hypermetabolic lesion compatible with tumor recurrence (Figure 1).

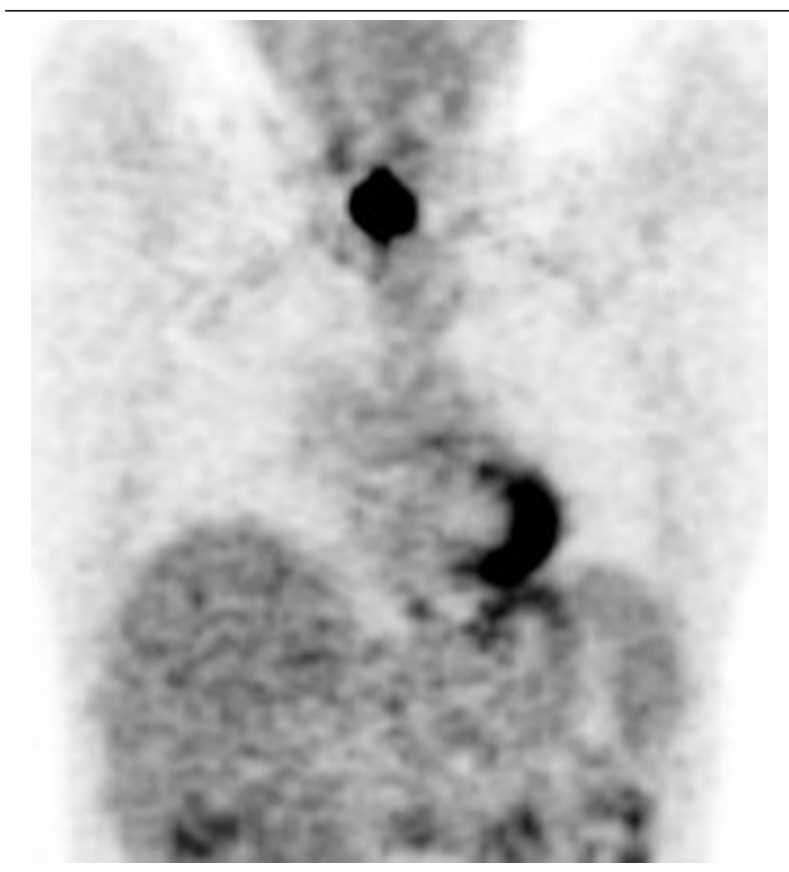

Figure 1.18F-FDG PET-CT Identified a Single Neck Hypermetabolic Lesion
Tumor resection was performed and invasion to larynx, trachea and esophagus was noticed intraoperatively. Histological examination revealed a $2.5 \mathrm{~cm}$ angiosarcoma, with positive surgical margins and immunohistochemistry staining showed the same features described above, including strong positivity result for CD31 with cytoplasmic and membranous pattern (Figure 2).

Figure 2. Histological Examination Revealed a 2,.5 cm Angiosarcoma, With Positive Surgical Margins
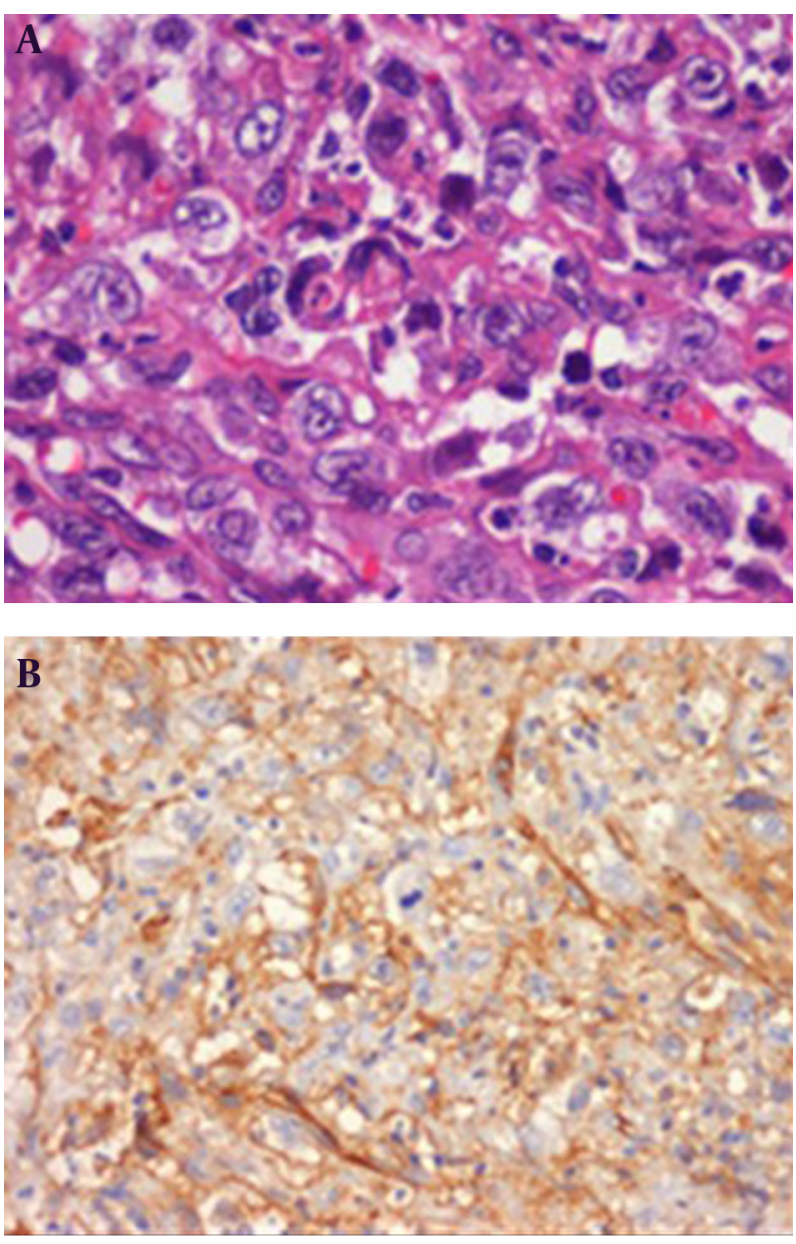

A, Tumor cells with vesicular nuclei with prominent nucleoli and abundant eosinophilic cytoplasm (H\&E staining); B, Strong positive result for CD31 with cytoplasmic and membranous pattern (Immunohistochemistry staining).

It was decided to treat the patient with local radiotherapy (60Gy), completed four years ago. The patient was kept under close surveillance at our institution, and about six years after the initial diagnosis, there was no clinical, analytical or imaging (neck CT, magnetic resonance imaging) evidence of tumor recurrence.

\section{Conclusions}

We described a rare case of thyroid angiosarcoma associated with a papillary microcarcinoma on the contralateral 
lobe in a patient living outside the Alpine region. A recent literature review by Kaur et al. (13) identified 48 cases of thyroid angiosarcoma worldwide. Since then, as far as we know, three more cases were published (14-16), and including one we described, there are a total of 52 patients with thyroid angiosarcoma in the literature. Generally, there was a favorable outcome despite initial local recurrence with invasion to adjacent organs. We are unaware of what might exactly influenced the patient's prognosis, but we believe that local radiotherapy may have an important role in the treatment of these types of lesions. Data available is insufficient to suggest the most suitable approach to this type of cases, but surgical treatment of head and neck angiosarcoma followed by radiotherapy improved patient's survival (13). However, there are some reports of patients treated with radiotherapy who did not respond to that modality of treatment $(5,6,17)$. We could not find any similar case of such a long disease free period, after radiation treatment. In areas not associated with endemic multinodular goiter, cases with higher than average survival have been reported (1), comparable to our patient's case. Good prognosis has been associated with absence of extra glandular extension (9), which was found in this case, but not changing the final outcome. The low incidence of these tumors in non-Alpine regions makes them difficult to diagnose and treat. Being so, we believe that multidisciplinary collaboration is essential in addressing this and other rare thyroid neoplasms.

\section{Authors' Contribution}

Study concept and design: Couto, Torres. Acquisition, Analysis and interpretation of data: Couto, Martins, Matos. Drafting of the manuscript: Couto. Critical revision of the manuscript for important intellectual content: Santos, Torres.

\section{References}

1. Petronella P, Scorzelli M, Luise R, Iannaci G, Sapere P, Ferretti M, et al. Primary thyroid angiosarcoma: an unusual localization.
World J Surg Oncol. 2012;10:73.

2. Eusebi V. Angiosarcoma. In: DeLellis RA, Lloyd RV, Heitz PU, Eng C editors. World health organisation classification of tumours Pathology and genetics tumours of endocrine organs.. Lyon: IARC Press; 2004. pp. 113-4.

3. Sniezek JC, Holtel M. Rare tumors of the thyroid gland. Otolaryngol Clin North Am. 2003;36(1):107-15.

4. Weiss SW, Goldblum JR. Malignant vascular tumors. In: Weiss SW, Goldblum JR editors. Enzinger and Weiss's Soft Tissue Tumors.. St Louis: Mosby; 2001. pp. 917-45.

5. Isa NM, James DT, Saw TH, Pennisi R, Gough I. Primary angiosarcoma of the thyroid gland with recurrence diagnosed by fine needle aspiration: a case report. Diagn Cytopathol. 2009;37(6):427-32.

6. Goh SG, Chuah KL, Goh HK, Chen YY. Two cases of epithelioid angiosarcoma involving the thyroid and a brief review of nonAlpine epithelioid angiosarcoma of the thyroid. Arch Pathol Lab Med. 2003;127(2):E70-3.

7. Kalitova P, Plzak J, Kodet R, Astl J. Angiosarcoma of the thyroid. Eur Arch Otorhinolaryngol. 2009;266(6):903-5.

8. Ryska A, Ludvikova M, Szepe P, Boor A. Epithelioid haemangiosarcoma of the thyroid gland. Report of six cases from a non-Alpine region. Histopathology. 2004;44(1):40-6.

9. Maiorana A, Collina G, Cesinaro AM, Fano RA, Eusebi V. Epithelioid angiosarcoma of the thyroid. Clinicopathological analysis of seven cases from non-Alpine areas. Virchows Arch. 1996;429(23):131-7.

10. Zouaidia F, Jahid A, Elouazzani H, Laraki L, Bernoussi Z, Mansouri F, et al. Angiosarcoma of the Thyroid Gland: A Case Report.J Med Cases. 2010;1(1):29-31.

11. Kim NR, Ko YH, Sung CO. A case of coexistent angiosarcoma and follicular carcinoma of the thyroid. J Korean Med Sci. 2003;18(6):908-13.

12. Yilmazlar T, Kirdak T, Adim S, Ozturk E, Yerci O. A case of hemangiosarcoma in thyroid with severe anemia due to bone marrow metastasis. Endocr J. 2005;52(1):57-9.

13. Kaur A, Didolkar MS, Thomas A. Angiosarcoma of the thyroid: a case report with review of the literature. Endocr Pathol. 2013;24(3):156-61.

14. Innaro N, Succurro E, Tomaino G, Arturi F. Nonalpine thyroid angiosarcoma in a patient with hashimoto thyroiditis. Case Rep Oncol Med. 2013;2013:901246.

15. Kefeli M, Mete O. An Unusual Malignant Thyroid Nodule: Coexistence of Epithelioid Angiosarcoma and Follicular Variant Papillary Thyroid Carcinoma. Endocr Pathol. 2013.

16. Gouveia P, Silva C, Magalhaes F, Santos C, Guerreiro E, Santos F, et al. Non-Alpine thyroid angiosarcoma. Int J Surg Case Rep. 2013;4(5):524-7.

17. Astl J, Duskova J, Limanova Z, Povysil C, Kuchynkova Z. Hemangiosarcoma of the Thyroid Gland. A case report. Neuro Endocrinol Lett. 2000;21(3):213-6. 